\title{
Prospective monitoring of the early treatment results of the Gl tract tumours at the Oncology Centre in Warsaw
}

\author{
Andrzej Rutkowski ${ }^{1}$, Tomasz Olesiński ${ }^{1}$, Piotr Surowski ${ }^{1}$, Michał Siwik², \\ Michał Potograbski², Magda Szałaj ${ }^{2}$
}

The current treatment of GI tract tumours is based to a large degree on the combined use of varied types of therapy: surgery, chemotherapy, irradiation. Severe post-operative complications may significantly affect the possibilities of application of non-surgical methods of treatment. Therefore, monitoring the early results of surgical treatment is extremely crucial to ensuring the appropriate necessary follow up and keep the risk of post-operative complications to a minimum. This paper describes the methodology of prospective collection of clinical data, which allows monitoring the quality of the surgical treatment of GI tumours on a current basis. On the basis of the data collected in the period from 2010 to 2015, the results of the analysis of three types of surgeries were presented (anterior resection of the anus, right hemicolectomy and a total gastric resection) and the actions taken on the basis of the annual report concerning the post-operative complication risks were described. Moreover, the examples of the application of prospectively collected clinical information for academic purposes were presented.

NOWOTWORY J Oncol 2016; 66, 6: 433-439

Key words: digestive surgery, postoperative complications, surgical quality

\section{Introduction}

Thanks to the introduction of new programmes of multidrug chemotherapy or molecularly directed (targeted) variants, a significant improvement in the treatment results of the majority of Gl tract tumours has been observed within the last 30 years. A good example here can be the prolongation of the overall survival period (OS) of the patients in the $4^{\text {th }}$ stage of a colorectal tumour of more than double [1]. The treatment of a patient is on the basis of the combined application of many therapies (surgery, irradiation, chemotherapy), and one must be aware of the relationship between the early results of each of the methods applied. Thus, for example, the significant toxicity of chemotherapy or neoadjuvant radiotherapy may affect the possibilities of surgical treatment. On the other hand, severe post-operative complications may render the continuation of treatment based on chemotherapy, impossible. This fact gains particular importance in cases where a surgery has a palliative character (e.g. the resection of a primary tumour in patients with non-resectable distant metastases which may be treated with chemotherapy). Given the above, it is necessary to monitor the quality of surgical treatment, measured not only with the scope of the lymphadenectomy performed (e.g. colorectal cancer, gastric cancer), the length of the resection margin (e.g. anal cancer, oesophageal cancer), the rate of the microscopically non-radical surgeries (R1 resection) or the surgeries complicated with iatrogenic perforation of the tumour; also the rate of post-surgical complications, especially those which prolong the duration of the hospital stay and/or require lengthy treatment. Permanent prospective supervision of the early results of surgical treatment, measured with a percentage of postoperative complications, also gives the possibility of correcting errors or shortcomings in the surgical technique and/or

${ }^{1}$ Department of Oncological Gastroenterology, Maria Skłodowska-Curie Memorial Cancer Center and Institute of Oncology, Warszawa, Poland

The Faulty of Medicine, Medical University of Warsaw, Warszawa, Poland 
in the perioperative treatment. The objective of this work is to present the methods of monitoring the early results of surgical treatment of patients with GI tract cancers treated at the Surgical Department of the Oncological Gastroenterology Clinic (before 2013: the Clinic of the Gastrointestinal Tract Tumours) and to present the benefits brought by the systematic prospective collection of clinical data.

\section{The methodology of data collection and reporting the results Database Chart}

Clinical data are collected in the Database Chart [To obtain the original contact the author]. The Chart is filled in by the doctor in charge of the case upon discharge from the ward. The chart contains five sections:

Section A - contains demographic data allowing for the identification of a patient, the diagnosis of the primary disease (which is an indication for the surgery) and the diagnosis of non-oncological comorbidities (which might potentially affect the outcome of surgical treatment), the data concerning synchronic or metachronic coexistence of other cancers (which might only have a potential effect on the outcome of the surgical treatment). Moreover this section contains data concerning the symptoms of infection diagnosed before the surgery and basic clinical data concerning nutritional status.

Section B - concerns the methods of pre-operative treatment. This section is filled only in a situation when a patient has received neoadjuvant therapy. Two basic pre-operative radiotherapy protocols have been distinguished: "short"irradiation, i.e. $5 \times 5$ Gy and "long" irradiation - classical radiotherapy. The above irradiation protocols concern patients suffering from rectal cancer. In the cases of a different cancer diagnosis (e.g. oesophageal cancer), the field of "long irradiation" should be ticked, which is the standard method of irradiating patients with a specific diagnosis. Moreover, this section of the chart contains information on pre-operative chemotherapy (provided that it was administered), with a requirement to specify the cytostatic agents which were used. The final paragraph of this section specifies the duration time period (the number of days) between the end of irradiation therapy and surgery.

Section C - describes the manner of surgical treatment. Apart from the date of the surgery, it contains the information concerning the mode of surgery, differentiating between elective and urgent modes, and the surgeries performed in an urgent mode (as per the definition contained in the regulations of the Ministry of Justice and Social (are) are described in the chart as emergency mode surgeries. It is necessary to specify the indications for a surgery in an urgent (emergency) mode. Apart from the name of the surgery performed, the surgeon specifies the method of reconstruction of the Gl tract (provided that it was reconstructed) and/or the method of reconstructing the defect in the abdominal integuments if some artificial material was used (e.g. fascial mesh). The character of the surgery is also stated: a radical surgery in the opinion of the surgeon, a palliative surgery, other type of surgery (e.g. diagnostic laparotomy with harvesting tissue specimens). In cases when the macroscopic features of dissemination, or a non-resectable primary tumour or local recurrence is found perioperatively and the procedure is then limited to harvesting specimens, the surgery is then defined as a palliative one. Also the cases of perioperative complications are recorded, such as: bleeding, infection of the peritoneal cavity, perforation of the intestine/tumour, iatrogenic spleen damage. Perioperative bleeding is defined as every case in which, as a result of blood loss into the surgical field, a drop in arterial pressure occurs and/or it is necessary to perform a perioperative blood transfusion or the blood loss exceeds $300 \mathrm{~mL}$ of blood extravasated into the surgical field and then suctioned. Perioperative bleeding also comprises cases in which it is necessary to tampon the surgical field as a result of the lack of possibilities of stopping the bleeding with electro-coagulation, ligation, underpinning or any other haemostatic measure (e.g. surgicel, spongostan, tissue glue). A perioperative complication is defined only as such cases of the perforation of the intestine/tumour which occurs during surgical procedures. This section also contains the information about the number and type of the blood products transfused, both before surgery and after. The final paragraphs of this section contain references to the surgeon (initials) and the duration of the surgery, from the moment of skin incision to the moment of placing the last stitch onto the integuments.

Section D - post-operative course. This section of the chart contains a description of all post-operative complications. The section specifies in detail 16 types of complications, but it allows also for annotating some other, non-specified complications. Separate paragraphs refer to the necessities of revision surgery, post-operative mortality (date of death) and the duration of hospital stay calculated from the date of surgery (surgery date: day 0 .). This section of the chart (similarly to all other sections) is filled in on the date of the patient's discharge and its intention is to specify early post-operative complications. Early complications are defined as all adverse events which occurr within 30 days of the date of surgery. If no post-operative complications were found on the date of hospital discharge, yet complications occurred within one month of the surgery (e.g. discharge on day 7 and on day 10 - the symptoms of an anastomotic leak), then earlier entries in the Chart are modified/corrected accordingly. Moreover, if the complication results in the necessity of one or more revision surgeries, the number of such procedures is recorded. It is important though, that in such cases no new chart is opened (even in the case when a 
patient requires a re-admission to hospital), but that previous entries in the existing chart are modified, including the duration of the hospital stay summing up the duration of the first stay with the consecutive one, resulting from the complications of the previous surgical treatment.

Section $\mathbf{E}$ - this is a section for additional information solely pertaining to the treatment of patients with rectal cancer.

The final paragraphs of the chart contain spaces for noting remarks which could complete the data already included in the chart, which, in the opinion of the doctor in charge of the case, could be significant for the outcomes of the treatment of patients with GI tract tumours (e.g. surgeries with the application of HIPEC - intraoperative hyperthermic intraperitoneal chemotherapy).

\section{Electronic databases}

All clinical data introduced into the chart are transferred to electronic databases, divided into sections concerning various tumour locations within the gastrointestinal tract. There are eight types of databases:

1. Rectal cancers,

2. Colon cancer,

3. Anal cancers,

4. Gastric cancers,

5. Oesophageal cancers,

6. Pancreatic cancers,

7. Bile ducts and gallbladder cancers,

8. Other cancers of the gastrointestinal tract.

With regards to the extensive character of the surgical procedures performed at the Surgical Ward of the Oncological Gastroenterology Clinic as well extending the scope of activity to new therapeutic procedures, four additional electronic databases have been created:

9. Other oncological diseases (not connected with the Gl tract),

10. Non-oncological diseases surgically treated,

11. Reconstructive surgeries,

12. Liver surgeries.

All the electronic databases are recorded in an Excel Program with the possibility of being transferred to other programs allowing for a detailed statistical analysis (e.g. SPSS programme). Only a limited number of staff have access to electronic databases (the Head of the Surgical Ward [TO], and the Co-coordinator of the Surgical Ward [AR]). The databases are password-protected.

\section{Verification of the quality of the collected data}

There are two levels of control for the collected data.

Level one - this is a verification of the data contained in the chart, which is performed upon the patient's discharge from hospital. The persons responsible for this level of verification is the Head of the Surgical Ward, who, upon signing the discharge summary, verifies the accuracy of the data collected. The source material then is the patient's information chart and the discharge summary.

Level two - entering data into the electronic database. The person responsible for the accuracy of entering this data into the databases is the Co-coordinator of the Surgical Ward, who is, at the same time, the administrator of the electronic databases. The source material is, in that case, the chart and the patient's information file as recorded in the ClinNet system.

\section{Reporting results}

The coordinator of the surgical team is responsible for the prospective monitoring of the early results of the surgical treatment. This is made possible thanks to analysis of the data contained in the electronic database. In the case of finding an increase in the rate of post-operative complications in a specific group of patients, the Coordinator informs the Head of the Surgical Ward and then the problem is discussed by the entire surgical team. Moreover, the Coordinator of the Surgical team prepares periodic reports (every 6 to 12 months concerning all electronic databases. These reports, in the form of a presentation, are presented to the whole surgical team. They concern both the current period and comparisons with the previous periods of the Clinic's activity.

\section{Benefits from a prospective evaluation of the quality of surgical treatment}

The discussion of all the benefits from the above described methods of prospective collection of clinical data would exceed the limits of this publication. However, two basic aspects must be distinguished here:

- clinical (current) which allows for monitoring early results of treatment and implementing at an appropriate moment (i.e. as soon as possible) the widely understood methods preventing the occurrence of post-operative complications;

- scientific (long-term) allowing for constructive research projects on the basis of the collected clinical material.To this end, the results of pathomorphology reports and long term follow-up results are attached to the collected clinical information.

\section{Examples of clinical application}

Prospective accumulation of clinical data allows for monitoring the percentage of the performed surgeries of a specific type and the related risk of post-operative complications on a current basis. The analysis of the three selected types of surgeries are discussed below.

\section{Anterior resection in patients with rectal adenocarcinoma}

Between July 2010 and December 2015, surgical treatment was performed in 405 patients of primary rectal cancer. 


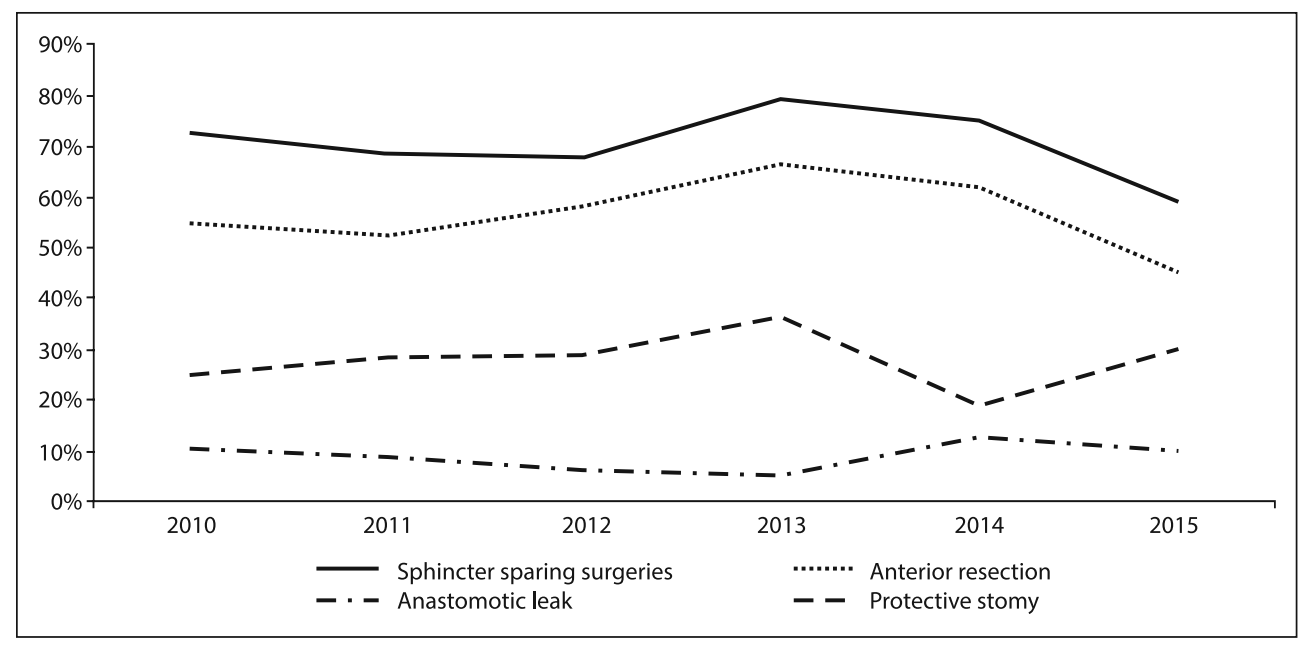

Figure 1. Anterior resection in primary rectal adenocarcinoma

Tumour resection was performed in 375 patients (93\%). In this group, the median distance between the edge of the anus and the inferior border of the tumour was $5 \mathrm{~cm}$. The total rate of resections saving the sphincters was $72.5 \%$, whereas the rate of surgeries with immediate reconstruction of the continuity of the alimentary tract $-60 \%$; (anterior resection: $n=219$, proctocolectomy with ileoanal anastomosis: $n=3$, local resection: $n=3$ ). The rate of symptomatic anastomotic leakages after the anterior resection varied between $5 \%$ and $13 \%$. At the same time, the rate of anastomoses protected with stomy was analysed (protective loop ileostomy) (Fig.1). Analysis of the database performed at the end of 2014 showed a slight decrease in the rate of sphincter-saving surgeries, i.e. mainly performed during anterior resections. Special attention was paid to the increase in symptomatic anastomotic leaks (to $12.8 \%$ in comparison with $5.2 \%$ in 2013). This was connected with a decrease in the number of surgeries in which the anastomosis was protected with a stomy: 19.1\% in 2014 in comparison with $36.2 \%$ in 2013 . As a result of this analysis, in 2015, a protective loop ileostomy was performed in 30\% of cases, and, consequently, the rate of anastomotic leaks was decreased. Some worrying data arrived in 2015, mainly with respect to significant decreases in sphincter saving surgeries and the proportion of anterior resections performed (59\% in comparison with 75\% in 2014 and $45.5 \%$ in comparison with $61.8 \%$ in 2014 , respectively). The data collected between January and June 2016 are indicative of the decrease in this worrying tendency, as during that period the rate of sphincter sparing surgeries was $74.3 \%$, whilst anterior resections - $62.9 \%$. The rate of anastomosis leaks is monitored on an ongoing basis.

\section{Right hemicolectomy in patients with adenocarcinoma} of the right-side of the colon

The analysis performed after the completion of the data concerning 2014 pointed to a growing tendency in the rate

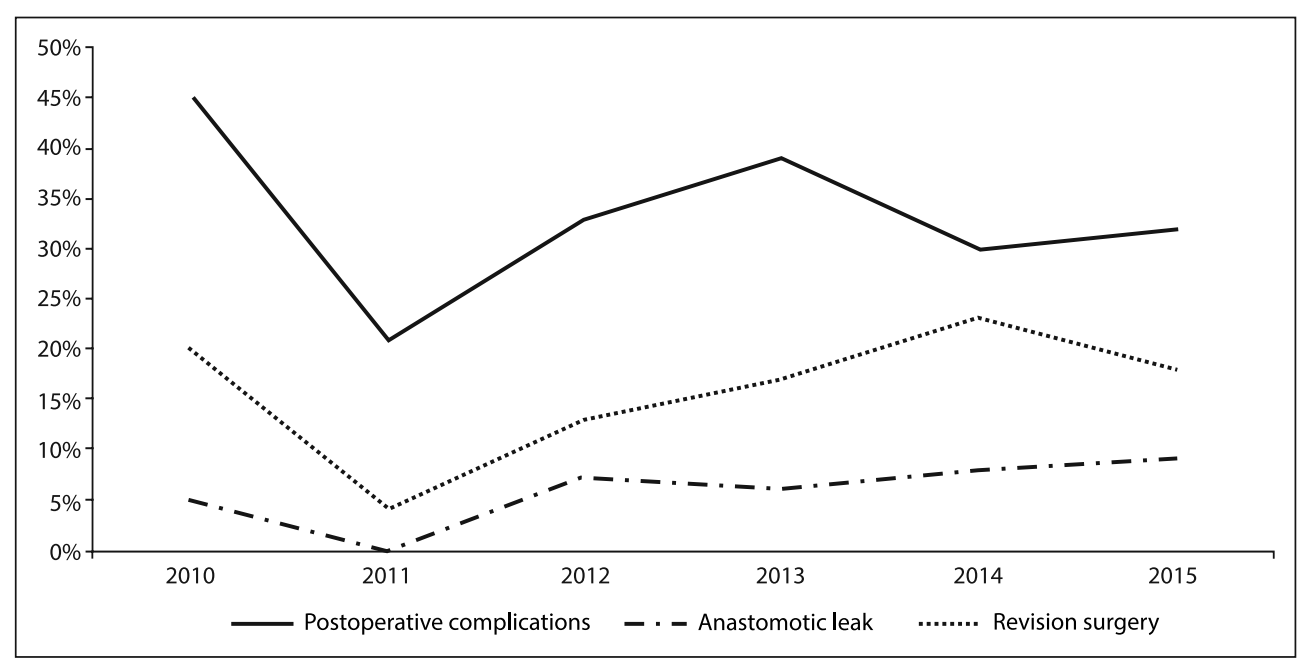

Figure 2. Right hemicolectomy in the tumour of the right-side of the colon 
of revision surgeries after right hemicolectomies (Fig. 2). A fact that raised particular concern was the high rate of anastomotic leaks (8\%). In the discussion of the surgeons' team, publications concerning the risk of complications were analysed with special attention paid to the technical aspects of making anastomoses after the resection of the right side of the colon (the type of staplers used, the height of the staples, additional sutures subsiding the staple lines etc.). Although clinical data for 2015 are indicative of a decrease in the rate of revision surgeries, the rate of anastomotic leaks remained at a comparatively high level (9\%). What is more, in 2015, 3 cases of deaths were observed in the post-operative period after a right hemicolectomy; this happened for the first time within a 6-year observation period. A detailed analysis of these cases showed that the direct cause of death was multi-organ dysfunction. All these those closing the duodenum) was observed. This worrying tendency was successfully halted in 2015. Analysis of complications after a total gastrectomy points to a stable, good situation in the early results of the surgical treatment of patients with gastric cancer. The rate of the complications, including the anastomotic leaks, remains at an acceptable level. What draws particular attention is the fact that the rate of revision surgeries is $9.7 \%$, whilst post-operative mortality remains below $1.5 \%$.

\section{Examples of scientific application}

Collected prospective clinical data were used for an analysis, the objective of which was the verification of the usefulness in clinical practice of the"risk scale of anastomotic leak after anterior resection", worked out on the basis of retrospective evaluation of 501 patients operated at the

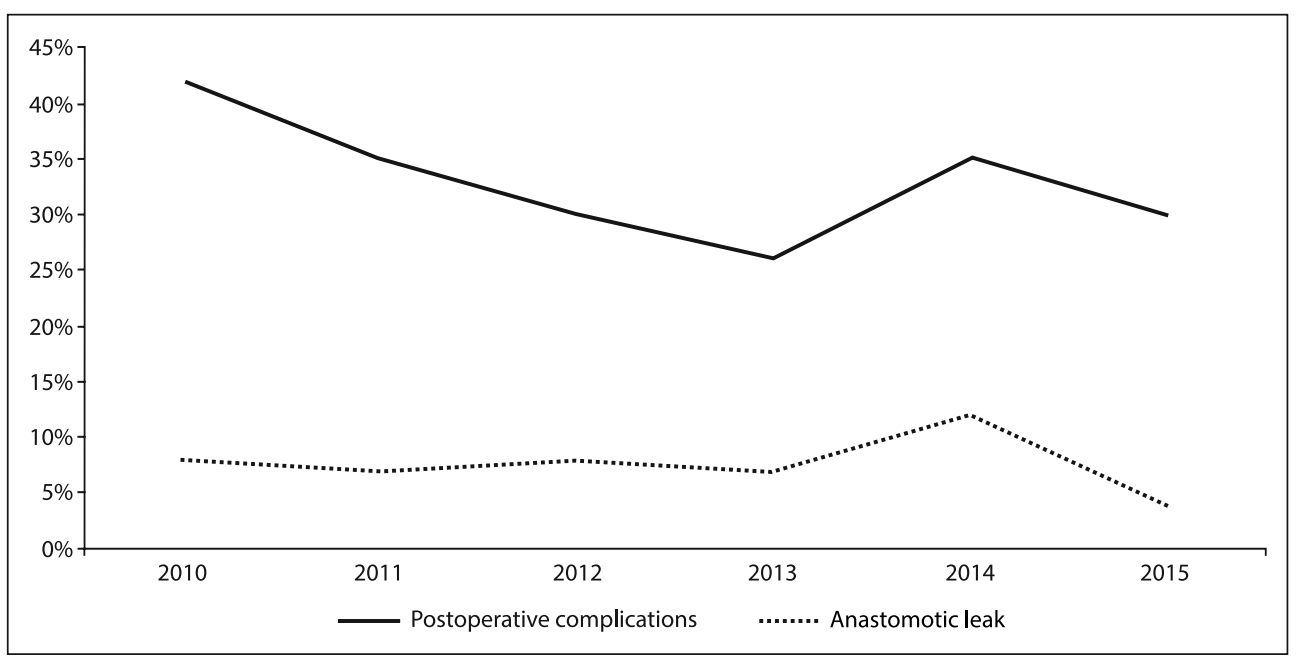

Figure 3. Total gastrectomy in gastric tumour

patients were re-operated on The indications for a revision surgery were: anastomotic leak $(n=1)$, wound dehiscence $(n=1)$ and post-operative bleeding $(n=1)$. The age of the patients was 77, 79 and 81 years respectively. All of them had a history of comorbidities: heart ischemic disease $(n=3)$, arterial hypertension $(n=3)$, diabetes $(n=2), \operatorname{COPD}(n=1)$. The conclusions which may be drawn from the analysis of these three cases comprise the necessity of a detailed assessment of the risk of severe post-operative complications in patients qualifying for such surgeries.

\section{Total gastric resection in patients with}

a primary gastric tumour

In the period between 2010 and 2013, a continual decrease in the rate of complications after a total gastrectomy was observed (Fig. 3). In 2014, an increase in the number of complications related to the anastomotic leak (mainly
Oncology Centre between 1996-2003 (Tab. I). The results of the analysis performed on the basis of the EBD "Colon Cancers" are presented in Table II. Depending on the score, three groups of patients were distinguished, where the rate of symptomatic anastomotic leak varied between $4.7 \%$ and $15.8 \%$. Protective colostomy seems to be necessary in patients with a high risk of anastomotic leak; in these cases the score of the points corresponding to some specific risk factors is from 5 to 8 . The analysis of these results carried out by a surgical team of the Clinic contributed to a decrease in the rate of anastomotic leaks in 2015, and, moreover, allowed the design of a multicentre prospective research project conducted under the auspices of the Polish Society of Surgical Oncology (research project:PSSO_01). Moreover, on the basis of EBD "Colon Cancers", there are prospective studies whose aim is to determine the factors which influence the quality of mesorectal excision, specifying the prog- 
Table I. The risk scale of the anastomotic leak after anterior resection — retrospective analysis of 501 cases of the patients operated on between $1996-2003$

\begin{tabular}{|c|c|c|}
\hline Category & Description & Score \\
\hline \multirow[t]{2}{*}{ Gender } & Female & 0 \\
\hline & Male & 1 \\
\hline \multirow[t]{2}{*}{ Pre-operative radiotherapy } & No & 0 \\
\hline & Yes & 1 \\
\hline \multirow[t]{3}{*}{ The anastomotic level from the edge of the rectum $(\mathrm{cm})$} & $>5$ & 0 \\
\hline & $>3-5$ & 2 \\
\hline & $\leq 3$ & 3 \\
\hline \multirow[t]{2}{*}{ Perioperative bleeding (blood loss > 200mL) } & No & 0 \\
\hline & Yes & 1 \\
\hline \multirow[t]{3}{*}{ Surgery duration (min.) } & $\leq 150$ & 0 \\
\hline & $>150-180$ & 1 \\
\hline & $>180$ & 2 \\
\hline
\end{tabular}

Table II. Anastomotic leak after anterior resection illustrated with the scoring scale in the prospective clinical material between 2010-2014

\begin{tabular}{|c|c|c|c|}
\hline \multirow[t]{2}{*}{ Anastomotic leak risk category } & \multirow{2}{*}{$\begin{array}{l}\text { The number and rate } \\
\text { of leaks (in total) }\end{array}$} & \multicolumn{2}{|c|}{ Rate of anastomotic leaks } \\
\hline & & Without protective stomy & With protective stomy \\
\hline $\begin{array}{l}\text { Low risk } \\
\text { Score: } 0-2 \\
n=86\end{array}$ & $4(4.7 \%)$ & $5.2 \%$ & $0 \%$ \\
\hline $\begin{array}{l}\text { Medium risk } \\
\text { Score: } 3-4 \\
n=72\end{array}$ & $6(8.3 \%)$ & $10.4 \%$ & $4.2 \%$ \\
\hline $\begin{array}{l}\text { High risk } \\
\text { Score: } 5-8 \\
n=38\end{array}$ & $6(15.8 \%)$ & $38.5 \%$ & $4.0 \%$ \\
\hline
\end{tabular}

nostic significance of the number of resected lymph nodes after irradiation and colon cancer resection or the factors allowing for an immediate reconstruction of the continuity of the alimentary tract after the resection of the colon cancer. Associating the data concerning the post-operative complications contained in EBD "Gastric Cancers" with the evaluation of the nutritional status of the patients in the NRS 2002 scale, allowed for an evaluation of the usefulness of this scale for the prognoses of the risk of post-operative complications. The results of this analysis were presented during the XVIII Congress of the Polish Society of Parenteral and Enteral Nutrition (POLSPEN) in 2016.

\section{Final remarks}

Analysis of single cases of the failures of surgical treatment, although extremely valuable, does not usually allow for drawing conclusions which might be applicable for a general population of patients. In analysing a single case, the score is either 0 or 1 (either there was an anastomotic leak -1 , or not -0 ). The analysis of a larger group of patients, as was shown with the example of the clinical application, allows for drawing more general conclusions. The most significant advantage of the prospective monitoring of the quality of treatment is the possibility of immediate reaction to a situation that might occur. The identification of the existing problem and its description, offers the chance to find a solution, not only in the available publications concerning the research in other centres, but also within a specific surgical team. A prospective collection of the clinical material also allows for minimising the risk of omitting any information that might be significant from a clinical point of view. In our experience, the appropriate processing of the data collected in the annual summaries of the team's work whose intention should not definitely be the internal competition, raises awareness of the existing complications as well as the necessity for continual improvement of professional qualifications. It is a generally known fact that in patients operated on for oesophageal, pancreatic, gastric or colorectal cancers, the experience of the surgeon remains one of the most significant prognostic factors. A population study carried out in Great Britain showed that mortality rate after a resection of oesophageal, gastric and pancreatic cancers was indirectly proportional to the number of cases previously operated 
on by the surgeon [2]. Similar observations concern the risk of post-operative complications in patients operated on for colorectal cancer [3], yet it is the individual experience of the surgeon and the entire surgical team is built not only from the number of the surgeries performed, but it comes also from a critical analysis of the results and the rate of the complications, perioperative mortality, the length of hospital stay etc. Therefore, it is not a surprise that an increasing number of data supports the argument that every surgeon should have the chance to critically monitor the results of the treatment they have conducted [4]. However, monitoring the early results of surgical treatment fits into a much wider context, i.e. a comprehensive evaluation of the quality of the oncological treatment. With this intention, in many European countries, some special programmes and registers were created, allowing for an ongoing assessment of the situation concerning the treatment of patients with specific cancers. An example to follow might be the Dutch Surgical Colorectal Audit (DSCA) [5]. Within the first two years of launching this programme, all Dutch hospitals were covered by it. The rate of compliance of reported cases in comparison with the Netherlands Cancer Registry [NCR] in 2010-2012 was 92-95\% respectively. This programme recorded data concerning the advancement stage of the disease, the methods of treatment and its results. Within three years of the audit, some improvement in the compliance of the diagnostic process and the treatment with the binding standards was observed and, at the same time, a significant decrease in the rate of postoperative complications, revision surgeries and mortality was recorded. Similar programmes have also been created in other countries [6]. Another example here might be the American National Cancer DataBase (NCDB), which collects information concerning, among others, demographical data (age, gender, race, region of residence), tumour characteristics (location, histological type, size, differentiation grade, advancement stage), method of treatment (type of surgery, chemotherapy and radiotherapy programme) and overall survival. Thanks to this information, it was observed that post-operative mortality after the resection of oesophageal cancer, measured within the period of 90 days post-operatively, is double as the mortality observed within the first 30 days post-operatively [7]. The authors of this article are aware that the introduction of a register in Poland, such as NCDB, or carrying out an audit like DSCA, would require the introduction of legislative changes, and first and foremost, would incur significant costs which our system cannot currently afford. That is why it would be even more valuable to introduce a uniform method of prospective data collection concerning the results of surgical treatment in other oncological centres. Even if this data is not concerned with all organ locations, it would still be worthwhile to unify the data concerning at least one cancer (e.g. gastric, cancer or colon cancer). It is not about competition between individual centres, but about a reliable and critical outlook on the actual situation concerning both early and late results of GI tract cancers. Once the clinical data, collected in a unified manner (as is the case in the presented example of EBD), are completed with the results contained in pathomorphological protocols and with remote observations on the basis of the follow-up carried out in every centre or on the basis of the National Cancer Register, both prospective and retrospective studies could be constructed, based on this significantly valuable material. Progress in combined treatment raises our hopes for an improvement in the oncological results, which, however, does not release a surgeon from the obligation of due diligence in their care of carrying out the best quality of surgery possible. After all, possible complications lead not only to an increase in treatment costs, but also, for some patients, complications mean losing the chance for receiving a combined treatment and, eventually, overcoming their cancer.

\section{Conflict of interest: none declared}

\section{Prof. Andrzej Rutkowski, MD, PhD}

Department of Oncological Gastroenterology Maria Sklodowska-Curie Memorial Cancer Center and Institute of Oncology

W.K. Roentgena 5

02-781 Warszawa, Poland

e-mail:az.rutkowski@onet.eu

Received: 29 Aug 2016

Accepted: 6 Oct 2016

\section{References}

1. Costi R, Leonardi F, Zanoni D et al. Palliative care and end-stage colorectal cancer management: the surgeron meets the oncologist. World J Gastroentero/ 2014; 20: 7602-7621.

2. Mamidanna R, Ni Z, Anderson $\mathrm{O}$ et al. Surgeon volume and cancer esophagectomy, gastrectomy and pancreatectomy: a population-based study in England. Ann Surg 2016; 263: 727-732.

3. Anwar $S$, Fraser $S$, Hill J. Surgical specialization and training - its relation to clinical outcome for colorectal surgery. J Eval Clin Pract 2012; 18: 5-11.

4. Pera $M$. The surgeon as a risk factor: the need for shared individual outcome reports and quality improvement strategies. Colorectal Dis 2016; 18: 533-534.

5. van Lersum NJ, Snijders HS, Henneman D et al. The Dutch surgical colorectal audit. Eur J Surg Oncol 2013; 39: 1063-1070.

6. Jegou D, Penninckx F, Vandendael T et al. Completeness and registration bias in PROCARE, a Belgian multidisciplinary project on cancer of the rectum with participation on a voluntary basis. Eur J Cancer 2015; 51: 1099-1108.

7. In $\mathrm{H}$, Palis BE, Merkow RP et al. Doubling of 30-day mortality by 90 days after esophagectomy: a critical measure of outcomes for quality improvement. Ann Surg 2016; 263: 286-291. 